\title{
Comparison of discrete Hodge star operators for surfaces
}

\author{
Mamdouh S. Mohamed ${ }^{\mathrm{a}, 1, *}$, Anil N. Hirani ${ }^{\mathrm{b}}$, Ravi Samtaney ${ }^{\mathrm{a}}$ \\ ${ }^{a}$ Mechanical Engineering, Physical Science and Engineering Division, King Abdullah University of Science and Technology, Jeddah, KSA \\ ${ }^{b}$ Department of Mathematics, University of Illinois at Urbana-Champaign, IL, USA
}

\begin{abstract}
We investigate the performance of various discrete Hodge star operators for discrete exterior calculus (DEC) using circumcentric and barycentric dual meshes. The performance is evaluated through the DEC solution of Darcy and incompressible Navier-Stokes flows over surfaces. While the circumcentric Hodge operators may be favorable due to their diagonal structure, the barycentric (geometric) and the Galerkin Hodge operators have the advantage of admitting arbitrary simplicial meshes. Numerical experiments reveal that the barycentric and the Galerkin Hodge operators retain the numerical convergence order attained through the circumcentric (diagonal) Hodge operators. Furthermore, when the barycentric or the Galerkin Hodge operators are employed, a super-convergence behavior is observed for the incompressible flow solution over unstructured simplicial surface meshes generated by successive subdivision of coarser meshes. Insofar as the computational cost is concerned, the Darcy flow solutions exhibit a moderate increase in the solution time when using the barycentric or the Galerkin Hodge operators due to a modest decrease in the linear system sparsity. On the other hand, for the incompressible flow simulations, both the solution time and the linear system sparsity do not change for either the circumcentric or the barycentric and the Galerkin Hodge operators.
\end{abstract}

Keywords: Discrete exterior calculus (DEC), Hodge star, Circumcentric dual, Barycentric dual

\section{Introduction}

Discrete exterior calculus (DEC) is a paradigm for the numerical solution of partial differential equations (PDEs) on simplicial meshes [1, 2]. A main advantage of DEC is the mimetic behavior of its discrete operators where they retain at the discrete level many of the identities/rules of their smooth counterparts. Over the past decade, DEC was used to numerically solve many physical problems including Darcy [3, 4] and incompressible flows $[5,6,7]$. The mimetic behavior of the DEC discrete operators generally results in superior conservation properties for DEC discretizations. There also exist other numerical methods to discretize vector PDEs on surfaces that are not based on DEC $[8,9,10,11,12]$.

The definition of most DEC operators requires a dual mesh related to the primal simplicial mesh. A common choice for the dual mesh is the circumcentric dual. The mutual orthogonality of the primal simplices and their circumcentric duals results in simple expressions for the discrete Hodge star operators. However, using the circumcentric dual limits DEC to only Delaunay simplicial meshes. In the case of non-Delaunay meshes, DEC implementations using the circumcentric dual along with the diagonal Hodge star definition yields incorrect numerical results [13]. Enabling DEC to handle arbitrary simplicial meshes is

\footnotetext{
${ }^{*}$ Corresponding author

Email addresses: mamdouh.mohamed@kaust.edu.sa (Mamdouh S. Mohamed), hirani@illinois.edu (Anil N. Hirani), ravi.samtaney@kaust .edu.sa (Ravi Samtaney)

${ }^{1}$ On leave, Department of Mechanical Design and Production, Faculty of Engineering, Cairo University, Giza, Egypt.
}

advantageous not only due to the extra flexibility in mesh generation but also due to significantly facilitating any subsequent local/adaptive mesh subdivision. This is important considering that successive subdivision of a Delaunay triangulation with obtuse-angled triangles would result in a non-Delaunay mesh.

An alternative choice for the dual mesh is the barycentric dual. Since the barycenter of a simplex always lies in its interior (unlike circumcenters), the dual barycentric cells are always non-overlapping for arbitrary simplicial meshes. However, the orthogonality between the primal and dual mesh objects that characterized the circumcentric dual is no longer valid for the barycentric dual. This implies that the DEC operators involving metrics may need to be redefined. For DEC applications over surface simplicial meshes, it becomes essential to redefine the Hodge star operator $*_{1}$ and its inverse $*_{1}^{-1}$. Two previous discrete definitions for Hodge operators on general simplicial meshes are the Galerkin [14] and the geometrical barycentric-based [15, 16] definitions. By barycentric-based definitions here we mean the Hodge star definitions that are applicable when a barycentric dual mesh is employed. Both the two aforementioned definitions result in a sparse (but nondiagonal) matrix representation for the Hodge star operator $*_{1}$. Such a non-diagonal matrix structure further complicates the representation of the inverse operator $*_{1}^{-1}$. However, it is worth pointing out that on surface simplicial meshes, the DEC discretization of common partial differential equations; e.g. Poisson equation and incompressible Navier-Stokes equations, may not require the inverse operator $*_{1}^{-1}$. An alternative approach for dealing with non-Delaunay meshes, for the specific case of a scalar Laplacian, is through the intrinsic Delaunay triangula- 
tion [17].

The purpose of this study is to evaluate the performance of the barycentric-based and the Galerkin Hodge operators compared with the circumcentric (diagonal) Hodge star operator. The comparison is carried out through the DEC discretization of Darcy and incompressible Navier-Stokes flows over surfaces. The main differences between the circumcentric versus the barycentric dual meshes are first addressed in section 2 . The definitions of various Hodge star operators are then provided in section 3. This is followed by numerical experiments for Darcy flow and incompressible Navier-Stokes equations in section 4, demonstrating the behavior of the considered Hodge star operators. The results demonstrate the numerical convergence as well as the linear system sparsity and the computational cost for the various operators definitions considered during the current study. The paper closes with conclusions emphasizing the main observations and discussing related future insights.

\section{Circumcentric versus barycentric dual meshes}

Consider a domain $\Omega$ approximated with the simplicial complex $K$. This paper focuses only on simplicial meshes over flat/curved surfaces, and therefore the domain is considered to have a dimension $N=2$. A $k$-simplex $\sigma^{k} \in K$ is defined by the nodes $v_{i}$ forming it as $\sigma^{k}=\left[v_{0}, \ldots, v_{k}\right]$. The definition of many DEC discrete operators requires the dual complex $\star K$ defined over the primal simplicial complex $K$. The dual to a primal simplex $\sigma^{k}$ is the $(N-k)$-cell denoted by $\star \sigma^{k} \in \star K$. The top dimensional $k$-simplices/cells are consistently oriented; e.g. counterclockwise, in all our examples here.

Defined on the primal and dual mesh complexes are the spaces of the discrete $k$-forms denoted by $C^{k}(K)$ and $D^{k}(\star K)$, respectively. For $N=2$, the spaces of the discrete forms are related via the discrete exterior derivative $d_{k}$ and the discrete Hodge star $*_{k}$ operators as shown in the following diagram

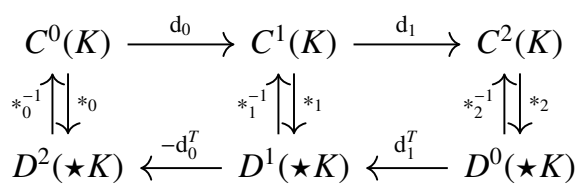

where the superscript $T$ indicates the matrix transpose.

A common choice for the dual complex is the circumcentric dual. This choice is motivated by the orthogonality between the primal and dual mesh objects, which simplifies the discrete Hodge star operators definitions. For example, for a smooth 1 -form $u$, the orthogonality of the primal edge $\sigma^{1}$ and its dual $\star \sigma^{1}$ makes the component of $u$ evaluated along $\sigma^{1}$ equal to the component of $* u$ evaluated along $\star \sigma^{1}$. This results globally in a diagonal matrix representation for the discrete Hodge star operator $*_{1}$ and also its inverse $*_{1}^{-1}$, simplifying various DEC computations.

Although the circumcentric duality yields this simplicity in the discrete Hodge star representation, this dual works correctly only on Delaunay meshes. Fig. 1a shows a sample non-Delaunay mesh. While the dual edges are, by the current convention, oriented $90^{\circ}$ counterclockwise with respect to their

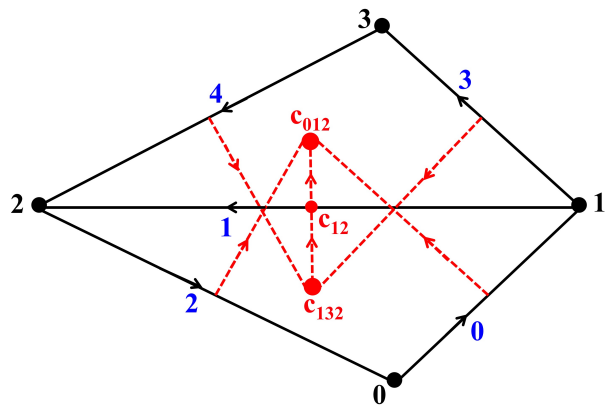

(a)

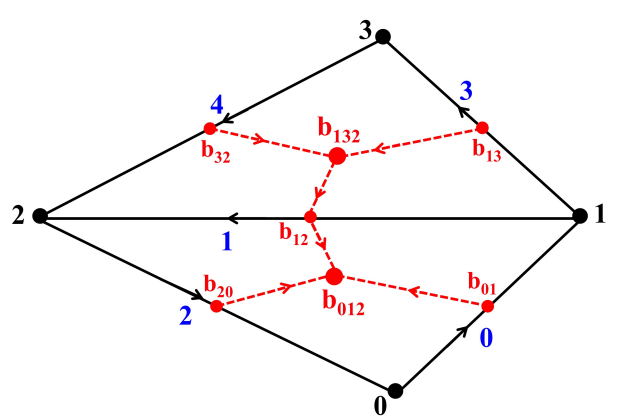

(b)

Figure 1: Sketch for a sample non-Delaunay mesh with: (a) the circumcentric dual, and (b) the barycentric dual. The primal mesh is in black color and the dual mesh is in red color. $\mathbf{c}_{i j k}$ and $\mathbf{b}_{i j k}$ are the circumcenter and barycenter of the triangle $\left[v_{i}, v_{j}, v_{k}\right]$, respectively. $\mathbf{c}_{i j}$ and $\mathbf{b}_{i j}$ are the circumcenter and barycenter of the edge $\left[v_{i}, v_{j}\right]$, respectively.

primal edges, the dual to the primal edge $\left[v_{1}, v_{2}\right]$ is oriented in the opposite direction. This is due to the circumcenters of the neighboring triangles being in the reversed order. According to the notation in [13], this implies that the dual edge $\star\left[v_{1}, v_{2}\right]$ has a negative volume. An additional concern is the construction of the dual areas. For a non-Delaunay mesh, the circumcentric dual areas overlap with some of the areas sectors having negatively-signed volumes. For example, for the mesh in Fig. $1 \mathrm{a}$, the areas dual to the primal nodes $v_{0}$ and $v_{3}$ are overlapping; i.e. $\star v_{0} \cap \star v_{3} \neq \emptyset$. In addition, for the area dual to the node $v_{1}$, the part of $\star v_{1}$ that does not overlap with $\star v_{0}$ and $\star v_{3}$ has a positive volume, while the part that overlaps with $\star v_{0}$ and $\star v_{3}$ has a negative volume, according to the volume calculation convention defined in [13]. Previous analysis showed that the DEC numerical solution of Poisson equation over a nonDelaunay mesh, using the diagonal definition of the Hodge star operator, leads to incorrect results [13].

An alternative choice for the dual mesh is the barycentric dual which is well defined on arbitrary simplicial meshes, as illustrated in Fig. 1b. The dual to a primal triangle is its barycenter, the dual to a primal edge is the kinked line connecting the barycenters of the two neighboring triangles through the barycenter of the primal edge itself, and the dual to a primal node is the polygonal area formed by the duals of the primal 


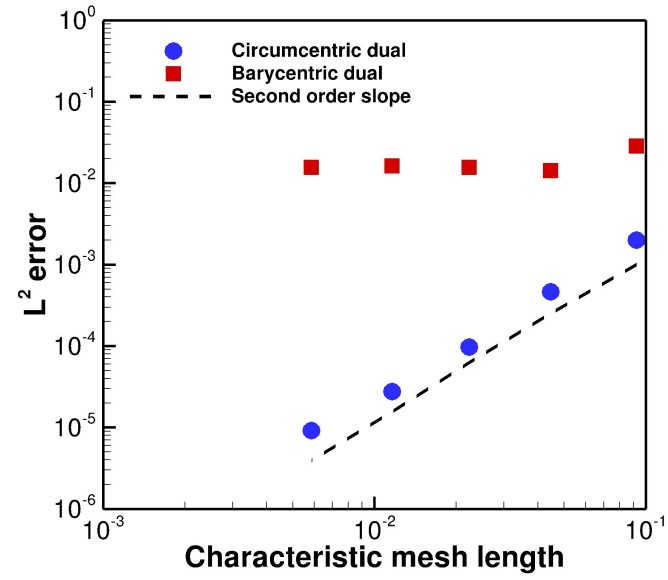

(a)

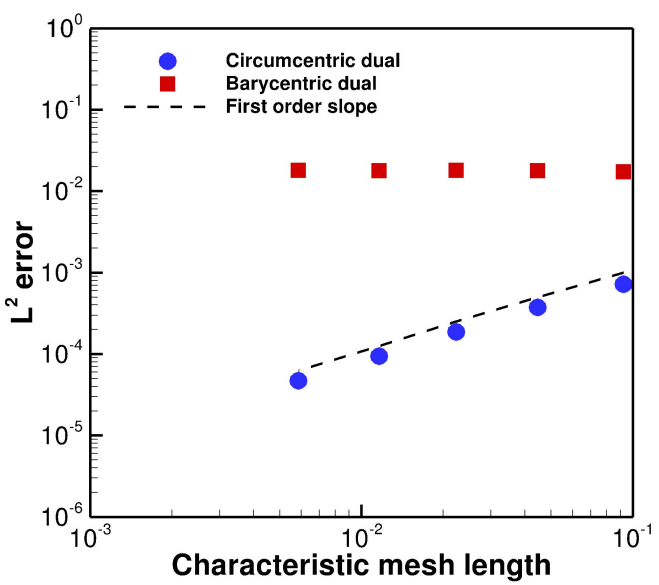

(b)

Figure 2: The convergence of the $L^{2}$ error (measuring the difference between the numerical and the exact analytical solutions) for (a) the 0-form $p$ in the Darcy flow problem, and (b) the 1-form $u$ in the incompressible Navier-Stokes equations. The solutions are computed using the diagonal definition of the discrete Hodge star operators for both the circumcentric and the barycentric duals. The problem formulations are discussed in Section 4 . The simulations are carried out over Delaunay meshes.

edges connected to the primal node. Since the barycenter of each triangle is always in its interior, the dual barycentric cells do not overlap for arbitrary simplicial meshes. On the other hand, it is evident from Fig. 1 that the main difference between the circumcentric and the barycentric duals is the lack of mutual orthogonality between the primal and dual edges in the case of the barycentric dual.

The lack of orthogonality between the primal edges and their barycentric duals invalidates the diagonal representation of some Hodge star operators. This can be illustrated through numerical DEC experiments using the diagonal Hodge star operator constructed using a barycentric dual mesh. Fig. 2 shows the $L^{2}$ norm error for the Darcy flow and the incompressible Navier-Stokes DEC solutions. More details regarding the problems formulations are provided in Section 4. It is observed in Fig. 2a that while the Darcy flow solution error using the circumcentric dual converges in a second order fashion as expected, the solution using the barycentric dual does not converge. A similar behavior is observed for the incompressible Navier-Stokes equations solution in Fig. 2b. While the $L^{2}$ error for the velocity 1-form converges as expected with a first order rate when using the circumcentric dual, the error does not converge when using the barycentric dual. This implies the existence of an error source that dominates the solution error, preventing its convergence with the mesh size. This error arises from the inappropriate diagonal definition of $*_{1}$ when a barycentric dual mesh is employed.

Two alternative discrete definitions for the Hodge operators are the Galerkin [14] and the geometrical barycentric-based $[15,16]$ Hodge operators. The lowest and highest order Hodge operators; i.e. $*_{0}$ and $*_{2}$ (and therefore their inverses $*_{0}^{-1}$ and $*_{2}^{-1}$ ), however retain their diagonal representation as $*_{k}=\frac{\left|\star \sigma^{k}\right|}{\left|\sigma^{k}\right|}$ for $k=0,2$, where |.| is the volume of the enclosed simplex/cell. The diagonal representation for these operators should be still valid since they actually represent a volume averaging process that should not in principle change due to the dual mesh selection. Indeed, these diagonal definitions are used in our numerical experiments in Section 4. Therefore, the barycentricbased and the Galerkin definitions are different here only for the Hodge operator $*_{1}$. In the next section, various definitions for $*_{1}$ are discussed in more detail.

\section{Hodge star definitions}

For a Delaunay simplicial mesh with a circumcentric dual, the primal-dual orthogonality allows a diagonal definition for the discrete Hodge operator as [1, 2]

$$
\left[*_{1}\right]_{i i}^{C}=\frac{\left|\star \sigma_{i}^{1}\right|}{\left|\sigma_{i}^{1}\right|} .
$$

As mentioned earlier, the diagonal structure of $*_{1}$ simplifies the discrete representation of the inverse operator $*_{1}^{-1}$, which is also diagonal.

For a general simplicial mesh, another discrete definition of the Hodge operator is based on Whitney forms [18], which do not require a dual mesh for their definition. For surface simplicial meshes; i.e. $N=2$, the Whitney 1 -form corresponding to the primal edge $\sigma^{1}=\left[v_{i}, v_{j}\right]$ defined over the primal triangle $\sigma^{2}=\left[v_{i}, v_{j}, v_{k}\right]$ is defined as

$$
W_{\left[v_{i}, v_{j}\right]}^{\left(\sigma^{2}\right)}=\mu_{i} \mathrm{~d} \mu_{j}-\mu_{j} \mathrm{~d} \mu_{i},
$$

with $\mu_{i}$ being the barycentric coordinate corresponding to the node $v_{i}$, and $\mathrm{d} \mu_{i}$ is its exterior derivative. Based on Whitney forms, the Galerkin Hodge star operator is defined as [14]

$$
\left[*_{1}\right]_{i j}^{G}=\int_{\sigma^{2}}\left\langle W_{\sigma_{i}^{1}}^{\left(\sigma^{2}\right)}, W_{\sigma_{j}^{1}}^{\left(\sigma^{2}\right)}\right\rangle \alpha
$$


where $\langle.,$.$\rangle denotes the interior product of the enclosed forms$ and $\alpha$ is the volume form on $\sigma^{2}$.

The Galerkin Hodge definition in Eq. (4) provides a symmetric sparse (but non-diagonal) representation for $*_{1}$. For the row corresponding to the primal edge $\left[v_{i}, v_{j}\right]$, the only nonzero entries in the Galerkin $*_{1}$ are at the columns corresponding to the primal edges belonging to the triangles sharing this primal edge $\left[v_{i}, v_{j}\right]$. Therefore, the number of nonzero entries in the Galerkin Hodge operator is almost five times compared with the circumcentric (diagonal) Hodge operator defined in Eq. (2). The effect of such decrease in the matrix sparsity is discussed in section 4.3. The evaluation of Eq. (4) requires numerical integration over each triangle using a sufficient number of quadrature points.

Another definition for $*_{1}$ is the geometrical barycentric-based Hodge operator defined in [16]. This definition is also based on Whitney forms but requires the use of the barycentric dual mesh. Consider a smooth 1-form $u$. We denote the form discretization on the primal edge $\sigma^{1}$ as $u_{\sigma^{1}}=\int_{\sigma^{1}} u$. Based on the values of the discrete 1 -form defined on the primal edges, it is required to get an estimation for the dual 1-form $w=* u$ discretized on a dual edge $\star \sigma^{1}$; i.e. $w_{\star \sigma^{1}}=\int_{\star \sigma^{1}} w$. The discretized primal 1-forms $u$ can be interpolated at a given point inside the triangle $\sigma^{2}$ as

$$
u^{\left(\sigma^{2}\right)}=\sum_{\sigma_{i}^{1}<\sigma^{2}} u_{\sigma_{i}^{1}} W_{\sigma_{i}^{1}}^{\left(\sigma^{2}\right)}
$$

where the summation is over all the faces $\sigma_{i}^{1}$ of the triangle $\sigma^{2}$.

Since $w=* u$, it is then possible to get an interpolated representation for the dual form $w$ at a given point inside the triangle $\sigma^{2}$ as

$$
\begin{aligned}
w^{\left(\sigma^{2}\right)}=* u^{\left(\sigma^{2}\right)} & =\sum_{\sigma_{i}^{1}<\sigma^{2}} u_{\sigma_{i}^{1}} * W_{\sigma_{i}^{1}}^{\left(\sigma^{2}\right)} \\
& =\sum_{\sigma_{i}^{1}<\sigma^{2}} u_{\sigma_{i}^{1}} W_{\sigma_{i}^{1}}^{\left(\sigma^{2}\right) *}
\end{aligned}
$$

where $W_{\sigma_{i}^{1}}^{\left(\sigma^{2}\right) *}=* W_{\sigma_{i}^{1}}^{\left(\sigma^{2}\right)}$ is the rotated Whitney forms due to the Hodge star action. Computationally, if a Whitney form was evaluated at a given point as $W_{\sigma_{i}^{1}}^{\left(\sigma^{2}\right)}=a \mathrm{~d} x+b \mathrm{~d} y$, then $W_{\sigma_{i}^{1}}^{\left(\sigma^{2}\right) *}=$ $-b \mathrm{~d} x+a \mathrm{~d} y$.

Eq. (6) provides an approximation of the smooth dual 1form $w$ inside $\sigma^{2}$ as a function of the discrete primal 1-forms $u_{\sigma_{i}^{1}}$. Such a representation when applied to the part of the dual edge $\star \sigma^{1}$ residing inside $\sigma^{2}$ would provide a discrete approximation for $w$ on that part of the dual edge. By repeating the same process on the other triangle sharing the dual edge $\star \sigma^{1}$, this provides an estimation for $w\left(\star \sigma^{1}\right)$. Denoting the part of the dual edge $\star \sigma_{i}^{1}$ that resides inside the triangle $\sigma_{k}^{2}$ as $\star \sigma_{i}^{1} \cap \sigma_{k}^{2}$, the evaluation of the dual form $w$ on the vector representing the dual edge $\star \sigma_{i}^{1}$ can be represented as

$$
w_{\star \sigma_{i}^{1}}=\sum_{\sigma_{k}^{2}} \sum_{\sigma_{j}^{1}<\sigma_{k}^{2}} u_{\sigma_{j}^{1}} W_{\sigma_{j}^{1}}^{\left(\sigma_{k}^{2}\right) *}\left(\star \sigma_{i}^{1} \cap \sigma_{k}^{2}\right),
$$

where the first summation is over the triangles $\sigma_{k}^{2}$ sharing the edge $\sigma_{i}^{1}$, and the second summation is over the faces of each triangle. The Whitney forms in Eq. (7) are considered here to be evaluated at the barycenter of $\sigma_{k}^{2}$.

The summed quantities in Eq. (7) are the discrete primal 1-forms multiplied by the rotated Whitney forms evaluated at the barycenter of $\sigma_{k}^{2}$ along parts of the barycentric dual edges. There exist a correspondence between the vector representations of the barycentric dual edges portions and the Whitney forms evaluated at the triangle's barycenter expressed as [16]

$$
\left\langle\star \sigma_{i}^{1} \cap \sigma_{k}^{2}, \mathbf{x}\right\rangle=\left|\sigma_{k}^{2}\right| W_{\sigma_{i}^{1}}^{\left(\sigma_{k}^{2}\right) *}(\mathbf{x}),
$$

where $\mathbf{x}$ is an arbitrary vector and the angle brackets here represent the inner product of the enclosed vectors. The above relation allows the barycentric-based geometric Hodge operator to be expressed as [16]

$$
\left[*_{1}\right]_{i j}^{B}=\left|\sigma^{2}\right|\left\langle W_{\sigma_{i}^{1}}^{\left(\sigma^{2}\right) *}, W_{\sigma_{j}^{1}}^{\left(\sigma^{2}\right) *}\right\rangle .
$$

Since $\left\langle W_{\sigma_{i}^{1}}^{\left(\sigma^{2}\right) *}, W_{\sigma_{j}^{1}}^{\left(\sigma^{2}\right) *}\right\rangle=\left\langle W_{\sigma_{i}^{1}}^{\left(\sigma^{2}\right)}, W_{\sigma_{j}^{1}}^{\left(\sigma^{2}\right)}\right\rangle$, the barycentric-based geometric Hodge definition is then the special case of the Galerkin Hodge definition in Eq. (4) when the integration is approximated by a one point quadrature with evaluation only at the triangle barycenter. In what follows, we denote the barycentric-based geometric Hodge operator in Eq. (9) as the barycentric Hodge star. The barycentric Hodge operator is symmetric and has exactly the same sparsity structure as the Galerkin Hodge star.

Due to the sparse, but non-diagonal, nature of the barycentric $*_{1}$ operator, a sparse representation for the inverse operator $*_{1}^{-1}$ is not yet known. In principle, a similar concept can be followed to develop a separate definition for $*_{1}^{-1}$ by interpolating the dual 1 -forms over the two dual areas sharing the primal edge, apply the Hodge star to the interpolated 1-forms, and then applying them on each portion of the primal edge. However, the theoretical machinery required to carry out such an interpolation has not been developed yet. For example, although it is currently possible to interpolate 0 -forms inside convex polygons [19], the interpolation of 1-forms inside such convex polygons is not amenable yet. Such an interpolation is even more challenging recalling that the barycentric dual edges are kinked lines and the dual areas may be non-convex. In case a discrete definition for $*_{1}^{-1}$ could be developed following the above approach, such a definition, along with the above barycentric definition for $*_{1}$ may not satisfy the Hodge star identity $* * u=(-1)^{k(N-k)} u$. In such a case, it may not be possible to use both $*_{1}$ and $*_{1}^{-1}$ operators simultaneously in solving a given partial differential equation. However, each operator might be used separately to solve a system of equations that does not include its discrete inverse operator.

For a three-dimensional (3D) simplicial mesh case, there exist Galerkin and barycentric discrete definitions for the $*_{2}$ operator [16]. Both definitions will result in a discrete sparse definition for $*_{2}$ accounting for the 2 -forms defined on the primal triangles that belong to tetrahedra sharing the dual edge. The development of a barycentric-based sparse definition for the inverse Hodge star operators; viz. $*_{1}^{-1}$ and $*_{2}^{-1}$, is currently, however, beyond reach. The operator $*_{1}^{-1}$ is of a special importance 
since it is essential for the 3D DEC solution of incompressible flows.

In spite of the above mentioned limitations, there exist many important physical problems whose numerical solution over surfaces only requires a definition for $*_{1}$. Examples for these problems are the Darcy flow and the incompressible NavierStokes equations. In addition, Eqs. (8) and (9) provide a potential path for studying convergence of DEC in relation to the lowest order finite element exterior calculus [20], since the barycentric Hodge star can be treated as a quadrature approximation of the Galerkin Hodge star. In the following section, the behavior of the Galerkin and the barycentric Hodge star operators is benchmarked against the circumcentric Hodge operator through the solution of the Darcy flow and the incompressible Navier-Stokes equations.

\section{Results and discussion}

Convergence tests are carried out for Darcy flow and incompressible Navier-Stokes equations solutions in 2D. The tests characterize the behavior of the Galerkin and the barycentric Hodge star operators versus the circumcentric (diagonal) Hodge operator. The implemented numerical experiments are for test cases with known analytical solutions, aiming to quantitatively demonstrate the differences between various Hodge star operators. The problem formulation and implementation details are briefly presented here for each problem. The reader may refer to [3] and [7] for more details regarding the DEC solution of Darcy flow and incompressible Navier-Stokes equations, respectively.

Four mesh types are used in the numerical experiments. First is a group of five Delaunay meshes created independently, where the number of the triangular elements in any finer mesh is almost four times the number of triangular elements in the coarser mesh. Second is a sequence of non-Delaunay meshes created by sequential subdivision of the coarsest non-Delaunay mesh. The subdivision is carried out by introducing the new nodes at the center points of the existing mesh edges. Next is a group of structured-triangular meshes consisting of isosceles right triangles. Lastly is a group of highly irregular nonDelaunay meshes generated by distorting the first group of Delaunay meshes to increase the triangles aspect ratios. The above mesh groups are generated for a unit square simulation domain. The number of triangular elements for the coarsest mesh in each group is 740, 890, 722 and 740 for the Delaunay, sequentially divided, structured-triangular and distorted non-Delaunay meshes, respectively. The maximum aspect ratio, defined as the ratio between the circumradius and the inradius, is approximately 3.7, 3.8, 2.4 and 26.0, respectively. Fig. 3 shows sample meshes from the four mesh groups. During the convergence analysis, the characteristic measure of the mesh length is the maximum primal edge length. Eqs. (2), (4) and (9) are used to define the circumcentric, Galerkin and the barycentric Hodge star operator $*_{1}$, respectively. The integration of the Galerkin Hodge star operator is carried out over each triangle using 3 quadrature points. For the $*_{0}, *_{0}^{-1}, *_{2}$ and $*_{2}^{-1}$ operators, the diagonal representation $*_{k}=\frac{\left|\star \sigma^{k}\right|}{\left|\sigma^{k}\right|}$ for $k=0,2$ is used for both

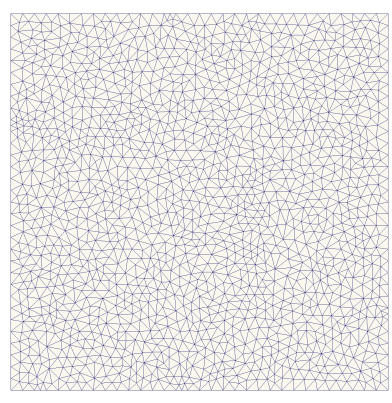

(a)

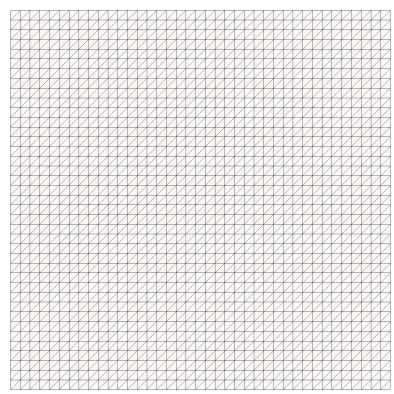

(c)

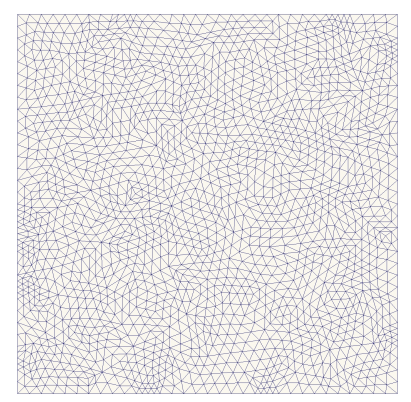

(b)

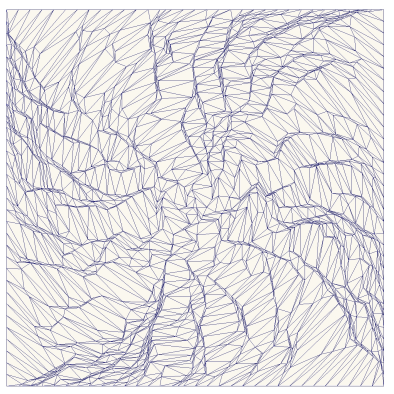

(d)
Figure 3: Sample meshes from the four types of triangulation used during the numerical experiments: (a) a Delaunay mesh, (b) a sequentially-divided non-Delaunay mesh, (c) a structured-triangular mesh, and (d) a distorted nonDelaunay mesh. The meshes shown in the figure are the second coarsest meshes in each group.

the circumcentric and the barycentric dual meshes, where the barycentric dual mesh is used along with both the Galerkin and the barycentric $*_{1}$ operators.

\subsection{Darcy flow}

The governing equations for Darcy flow, in the absence of body forces, are expressed in vector calculus notation as

$$
\begin{aligned}
& \mathbf{v}-\nabla p=0 \quad \text { in } \Omega, \\
& \nabla . \mathbf{v}=\phi \quad \text { in } \Omega \text {, } \\
& \mathbf{v . n}=\psi \quad \text { on } \partial \Omega,
\end{aligned}
$$

where $\mathbf{v}$ is the velocity vector and $p$ is the pressure. In exterior calculus notation, the Darcy flow equations can be expressed in terms of the velocity 1 -form $\mathbf{v}^{b}$ and the pressure 0 -form $p$ as [3]

$$
\begin{array}{ll}
\mathbf{v}^{\mathrm{b}}-\mathrm{d} p=0 & \text { in } \Omega, \\
* \mathrm{~d} * \mathbf{v}^{\mathrm{b}}=\phi & \text { in } \Omega .
\end{array}
$$

By choosing to define the pressure 0 -form $p$ on the primal nodes and the velocity 1 -form $\mathbf{v}^{b}$ on the primal edges, the discrete governing equations become

$$
\begin{aligned}
v-\mathrm{d}_{0} p & =0, \\
*_{0}^{-1}\left[-\mathrm{d}_{0}^{T}\right] *_{1} v & =\phi-*_{0}^{-1} \mathrm{~d}_{b} u,
\end{aligned}
$$

where $v$ is the primal velocity 1 -form. The operator $\mathrm{d}_{b}$ complements the action of the $\left[-\mathrm{d}_{0}^{T}\right]$ operator for the dual areas whose 
boundary includes primal edges [7]. The primal 1-form $u$ accounts for the flux across the boundary primal edges. Therefore, the Neumann boundary conditions are accounted for by the force vector $*_{0}^{-1} \mathrm{~d}_{b} u$. This is very similar to the finite element formulation of the scalar Poisson equation where Neumann boundary conditions are treated as natural boundary conditions represented by a force vector.

An analytical solution for the pressure field that satisfies Eqs. (10) is $p=\cos (\pi x) \cos (\pi y)$. For a unit square simulation domain, the Neumann boundary condition then becomes $\mathbf{v} . \mathbf{n}=0$; i.e. $u=0$ on $\partial \Omega$. The discrete system of equations to be solved is then

$$
\left[\begin{array}{cc}
I & -\mathrm{d}_{0} \\
*_{0}^{-1}\left[-\mathrm{d}_{0}^{T}\right] *_{1} & 0
\end{array}\right]\left[\begin{array}{c}
v \\
p
\end{array}\right]=\left[\begin{array}{l}
0 \\
\phi
\end{array}\right],
$$

where the pressure $p$ is specified at one interior node in order to get a unique solution for the system. Eq. (13) can be symmetrized by multiplying the first row by $*_{1}$ and the bottom row by $*_{0}$. This would however result in replacing the diagonal $I$ matrix by a sparse, but non-diagonal, $*_{1}$ in the barycentric and the Galerkin Hodge cases.

The above Darcy flow problem is solved over both the Delaunay and non-Delaunay meshes. For the Delaunay meshes, the problem is solved using the circumcentric, Galerkin and the barycentric Hodge star definitions. For the non-Delaunay meshes, the problem is solved using only the Galerkin and the barycentric Hodge star definitions. The $L^{2}$ norm error for the pressure field is calculated and plotted versus the maximum length of the primal edges for each mesh, as shown in Fig. 4. It is observed that for all the implemented test cases, the $L^{2}$ error converges in a second order fashion as expected. Even for the highly distorted non-Delaunay meshes, both the barycentric and the Galerkin Hodge operators reproduce the expected convergence rate. There is a very small difference, in terms of the solution $L^{2}$ error, when using the circumcentric versus the barycentric Hodge operators over the Delaunay meshes. The results for the Galerkin Hodge operator are found to be almost identical to those of the barycentric Hodge operator for all conducted test cases. It is worth noting that both the Galerkin and barycentric $*_{1}$ matrices do have the same sparsity structure but the individual entries in the matrices are different. Although the barycentric Hodge operator requires only one point integration (at the barycenter), it ultimately produces solutions similar to the Galerkin Hodge operator (using 3 integration points). This suggests that the barycentric Hodge operator is more computationally efficient than the Galerkin Hodge operator for the DEC Darcy flow solution. Both the barycentric and the Galerkin Hodge operators reproduce the convergence order attained via the circumcentric operator.

\subsection{Incompressible Navier-Stokes equations}

The equations governing the incompressible flow of a homogeneous fluid, with unit density and no body forces, over surfaces are the Navier-Stokes equations expressed as [7]

$$
\begin{aligned}
& \frac{\partial \mathbf{u}^{\mathrm{b}}}{\partial t}-v * \mathrm{~d} * \mathrm{~d} \mathbf{u}^{\mathrm{b}}+*\left(\mathbf{u}^{\mathrm{b}} \wedge * \mathrm{~d} \mathbf{u}^{\mathrm{b}}\right)+\mathrm{d} p^{d}=0, \\
& * \mathrm{~d} * \mathbf{u}^{\mathrm{b}}=0
\end{aligned}
$$

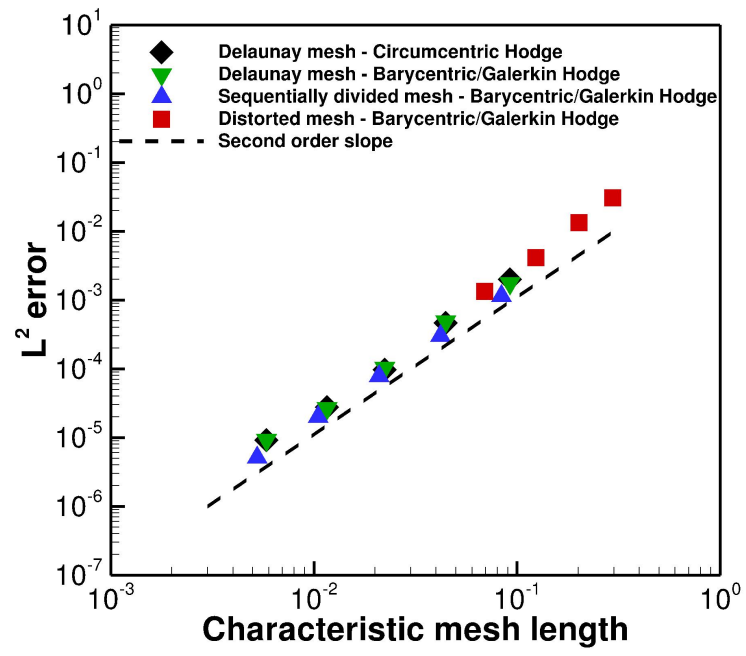

Figure 4: The numerical convergence of the pressure $L^{2}$ error (measuring the difference between the numerical and the exact analytical solutions) for the Darcy flow problem with Neumann boundary conditions. The results of the Galerkin and the barycentric Hodge star are identical, and therefore both are represented by the same symbols in the figure.

where $\mathbf{u}^{\mathrm{b}}$ is the velocity 1 -form, $p^{d}$ is the dynamic pressure 0 form defined as $p^{d}=p+\frac{1}{2}\left(\mathbf{u}^{\mathrm{b}}(\mathbf{u})\right)$, and $v$ is the kinematic viscosity. The DEC discretization of the momentum equation (14a) is

$$
\begin{aligned}
& \frac{u^{n+1}-u^{n}}{\Delta t}-v *_{1} \mathrm{~d}_{0} *_{0}^{-1}\left[\left[-\mathrm{d}_{0}^{T}\right] u+\mathrm{d}_{b} v\right] \\
& +*_{1} W_{v} *_{0}^{-1}\left[\left[-\mathrm{d}_{0}^{T}\right] u+\mathrm{d}_{b} v\right]+\mathrm{d}_{1}^{T} p^{d}=0,
\end{aligned}
$$

where $u$ is the dual velocity 1 -form and $v$ is the primal (tangential) velocity 1 -form. The matrix $W_{v}$, which contains the values of the tangential velocity 1-form $v$, represents the discrete wedge product of $v$ with the primal 0 -form $* \mathrm{~d} u$. A definition for this discrete primal-primal wedge product is given in [1]. The operator $\mathrm{d}_{b}$ again complements the action of $\left[-\mathrm{d}_{0}^{T}\right]$ on the dual areas whose boundary includes primal edges.

Taking the exterior derivative of Eq. (15) and substituting for the velocity dual 1 -form by $u=*_{1} \mathrm{~d}_{0} \psi$, the discrete system of equations to be solved is then

$$
\begin{array}{r}
\frac{1}{\Delta t}\left[-\mathrm{d}_{0}^{T}\right] *{ }_{1} \mathrm{~d}_{0} \psi^{n+1}-v\left[-\mathrm{d}_{0}^{T}\right] *{ }_{1} \mathrm{~d}_{0} *_{0}^{-1}\left[-\mathrm{d}_{0}^{T}\right] *{ }_{1} \mathrm{~d}_{0} \psi^{n+1} \\
+\left[-\mathrm{d}_{0}^{T}\right] *{ }_{1} W_{v}^{n} *_{0}^{-1}\left[-\mathrm{d}_{0}^{T}\right] *{ }_{1} \mathrm{~d}_{0} \psi^{n+1}=F,
\end{array}
$$

with the vector $F=\frac{1}{\Delta t}\left[-\mathrm{d}_{0}^{T}\right] u^{n}+v\left[-\mathrm{d}_{0}^{T}\right] *_{1} \mathrm{~d}_{0} *_{0}^{-1} \mathrm{~d}_{b} v^{n}-\left[-\mathrm{d}_{0}^{T}\right] *{ }_{1}$ $W_{v}^{n} *_{0}^{-1} \mathrm{~d}_{b} v^{n}$. The degrees of freedom in the above linear system are the stream function 0 -forms $\psi$ defined on the primal mesh nodes. Therefore, the resulting system is a sparse linear system of size equal to the number of the primal mesh nodes. The reader may refer to [7] for more details regarding the discretization.

The numerical convergence analysis for the incompressible Navier-Stokes equations is carried out through the implementation of the Poiseuille flow test case. For a unit square simulation domain, solid wall boundary conditions are applied on 
the top and bottom boundaries of the simulation domain, and a parabolic in/out flow conditions are imposed on the left/right boundaries. With the flow across all boundary primal edges to be known, the boundary conditions for the stream function $\psi$ can be easily set. The exact solution of the velocity vector field is $\mathbf{u}=(y(1-y), 0)$ for $v=1.0$. The $L^{2}$-error of the velocity 1 -form $u$ is calculated as $\left\|u^{\text {exact }}-u\right\|=\left[\sum_{\sigma^{1}} A_{s}\left(\frac{u^{\text {exact }}-u}{\left|\sigma^{1}\right|}\right)^{2}\right]^{1 / 2}$, where $u^{\text {exact }}$ is the integration of the exact velocity on the dual edge and $A_{s}$ is the support area for each primal edge $\sigma^{1}$. For the circumcentric dual mesh case, the support area is $A_{s}=\left|\sigma^{1}\right|\left|\star \sigma^{1}\right| / 2$.

The convergence of the $L^{2}$ error with the mesh size is shown in Fig. 5. For the structured-triangular meshes, a second order convergence rate is observed, with almost no difference in the $L^{2}$ error between the circumcentric versus the barycentric Hodge star. For the Delaunay mesh simulations, the $L^{2}$ error converges in a first order fashion, with the barycentric Hodge star solution error being slightly larger than that for circumcentric Hodge star. The barycentric Hodge star operator then successfully reproduces the convergence rate attained through the circumcentric Hodge operator for the Delaunay mesh test case. A first order convergence rate is also observed for the highly distorted non-Delaunay mesh simulations using both the barycentric and the Galerkin Hodge operators. Such convergence rates are in agreement with previous theoretical analysis by Nicolaides [21], for the covolume method using a circumcentric dual, showing that a second order convergence rate is attainable when the center point of each primal edge coincides with the center point of its dual edge. This condition is satisfied for the structured-triangular meshes with either the circumcentric or the barycentric dual meshes. For otherwise unstructured meshes, the error convergence is expected to have a first order rate, as shown in Fig. 5. The Galerkin Hodge results are again similar to the barycentric Hodge results for all conducted experiments.

For solutions over sequentially divided non-Delaunay meshes using the barycentric Hodge star, the error convergence rate is very close to a second order. The convergence rate increases with the mesh refinement, where the convergence rate between the first two coarser meshes is $\simeq 1.65$ and reaches $\simeq 1.85$ between the two finest meshes. On average, the slope of the line between the coarsest and finest mesh is $\simeq 1.77$. Such a convergence rate is more than what is usually expected for unstructured simplicial meshes. This observed super-convergence can be attributed to the sequential subdivision of the meshes. Fig. 6 shows two triangles that were subdivided by introducing new nodes at the primal edges midpoints, resulting in eight finer triangles. For the newly generated primal edges inside the coarser triangles; e.g. the edge $\left[v_{4}, v_{5}\right]$ in Fig. 6 , the midpoints of these primal edges coincide with their duals midpoints. This is the condition required to attain a second order convergence rate, as discussed earlier for the structured-triangular mesh case. Since this super-convergence condition is not satisfied for the finer edges that reside on the original coarse edges; e.g. the edge $\left[v_{3}, v_{6}\right]$, the convergence rate is still somewhat lower than second order. As more new interior edges are introduced dur-

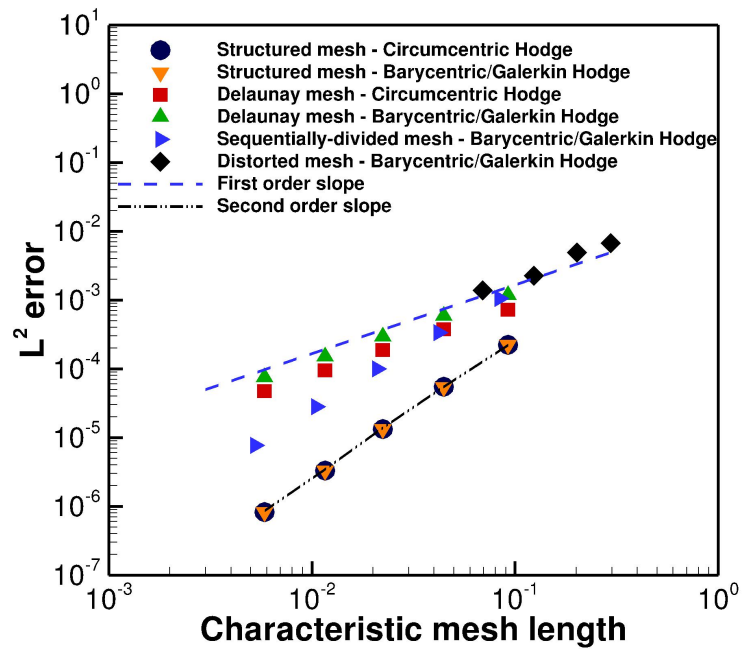

Figure 5: The numerical convergence of the $L^{2}$ error (measuring the difference between the numerical and the exact analytical solutions) of the velocity 1form $u$ for the Poiseuille flow test case. The results of the Galerkin and the barycentric Hodge star are identical, and therefore both are represented by the same symbols in the figure.

ing the sequential refinement, the convergence rate approaches second order. A similar super-convergence behavior was also observed for the Galerkin Hodge operator. It is interesting that the sequential subdivision of the unstructured meshes, which was a challenge when using the circumcentric Hodge star, became an advantage when using the barycentric and the Galerkin Hodge operators.

\subsection{Computational aspects}

One of the main concerns regarding the barycentric and the Galerkin Hodge operators, in addition to the difficulty of deducing a sparse inverse operator, would at first appear to be the computational cost. Both the Galerkin and the barycentric operator matrices have a number of nonzero entries almost five times as that for the circumcentric (diagonal) Hodge operator.

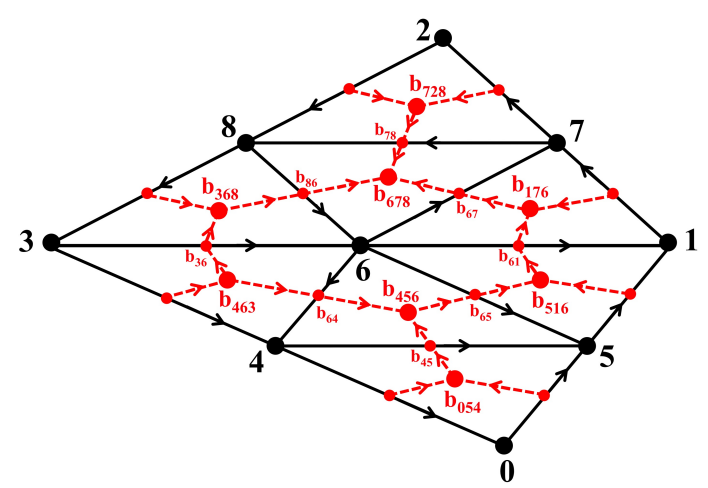

Figure 6: A sample simplicial mesh that was refined through subdivision. The subdivision is carried out by introducing the new nodes at the midpoints of the coarse mesh edges. 
One might then logically expect an increase in the linear system solution time. This section addresses these computational aspects by examining the matrix sparsity and solution time during Darcy and incompressible flow simulations. It is shown in this section that the additional computational cost when using the barycentric or the Galerkin Hodge star is minimal or non existent. The Darcy flow linear system is solved here using a biconjugate gradient solver, while a sparse LU decomposition solver is used to solve the incompressible Navier-Stokes linear system. The results presented below are implemented over the Delaunay mesh group.

Fig. 7a shows the number of nonzero entries in the system matrices for Darcy and incompressible flow problems when using the circumcentric and the barycentric Hodge operators. In regards to the Darcy flow problem, the number of nonzero entries increases on average by almost $40 \%$ when the barycentric Hodge is used. On the other hand, the incompressible flow problem do not exhibit any change in the number of nonzero entries in its system matrix when using either the circumcentric or the barycentric Hodge operators. This can be explained through Table 1, which lists the number of nonzero entries in some DEC matrices when using the circumcentric and the barycentric Hodge operators. The barycentric $*_{1}$ matrix has a number of nonzero entries almost five times as that for the circumcentric Hodge matrix. However, the difference in terms of the matrix $\left[*_{1} \mathrm{~d}_{0}\right]$ reduces to a factor of two, as shown in Table 1. When using the circumcentric Hodge operator, the nonzero entries in any column of the matrix $\left[*_{1} \mathrm{~d}_{0}\right]$, corresponding to a primal node, are in the rows corresponding to the primal edges connected to this node. In the barycentric Hodge operator case, the nonzero entries in any column of the matrix $\left[*_{1} d_{0}\right]$ would then be for all the edges belonging to the triangles sharing this node, which is almost twice as the number of edges connected to the node. The multiplication by the matrix $\mathrm{d}_{0}$, which includes a differentiation stencil, would then mitigate some of the lower sparsity effect of the barycentric Hodge matrix. Further multiplication by another exterior derivative matrix would completely eliminate the lower sparsity effect of the barycentric Hodge matrix due to the further increase in the effective differentiation stencil. This can be noticed in Table 1 where the number of nonzero entries in the matrix $\left[d_{0}^{T} *_{1} d_{0}\right]$ is identical for both the circumcentric and the barycentric Hodge operators.

\begin{tabular}{c|c|c} 
& Circumcentric Hodge & Barycentric Hodge \\
\hline$*_{1}$ & 1143 & 5583 \\
$\mathrm{~d}_{0}$ & 2286 & 2286 \\
$*_{1} \mathrm{~d}_{0}$ & 2286 & 4506 \\
$\mathrm{~d}_{0}^{T} *_{1} \mathrm{~d}_{0}$ & 2690 & 2690 \\
\hline
\end{tabular}

Table 1: The number of nonzero entries in some of the DEC matrices for both circumcentric and barycentric Hodge operators. The tabulated numbers correspond to a Delaunay mesh consisting of 404 nodes, 1143 edges and 740 triangles.

Accordingly, for the Darcy flow solution, the number of nonzero entries of the block matrix $\left[*_{0}^{-1}\left[-\mathrm{d}_{0}^{T}\right] *_{1}\right]$ in Eq. (13) increases by a factor of two when the barycentric Hodge operator is used. This ultimately leads to almost $40 \%$ increase in the number of nonzero entries in the whole linear system, as shown in Fig. 7a, and a corresponding slight increase in the linear system solution time, as shown in Fig. 7b. For the incompressible flow problem, since each Hodge matrix $*_{1}$ is multiplied twice by exterior derivative operators, as in Eq. (16), the sparsity of the resulting linear system does not change when using the circumcentric or the barycentric Hodge operator. Consequently, the linear system solution times do not vary, as shown in Fig. 7b. The above results suggest that the lower sparsity of the barycentric and the Galerkin Hodge operator matrices has generally a modest effect on the computational cost.

\section{Conclusions}

The paper presented a comparison between the circumcentric (diagonal) Hodge operator versus the Galerkin and the barycentric (geometric) Hodge operators on surface simplicial meshes. While the circumcentric Hodge operator works properly only on Delaunay meshes, the Galerkin and the barycentric Hodge operators admit arbitrary simplicial meshes. This provides more flexibility to the meshing process and facilitates any subsequent mesh subdivision, which would require edge flips or remeshing in case only Delaunay simplicial meshes were allowed.

The Galerkin Hodge operator definition is based on the integration of Whitney forms over the triangles. The barycentric Hodge operator is defined also through Whitney forms, and can be considered to correspond to a one-point quadrature version of the Galerkin Hodge operator with quadrature points at the triangles barycenters. Both definitions resulted in a sparse, non-diagonal, definition for the Hodge star operator $*_{1}$. Such a sparse representation complicates the development of a sparse barycentric or Galerkin definitions for the inverse operator $*_{1}^{-1}$. The barycentric and the Galerkin definitions for $*_{1}$ are, however, advantageous for many physical problems that do not require $*_{1}^{-1}$; e.g. the Poisson and incompressible Navier-Stokes equations. In regards to the three-dimensional case, although the existence of barycentric and Galerkin definitions for the $*_{1}$ and $*_{2}$ operators is known, it is not known yet how a sparse representation for $*_{1}^{-1}$ and $*_{2}^{-1}$ can be defined. The operator $*_{1}^{-1}$ is essential for the 3D DEC discretization of incompressible Navier-Stokes flows.

Numerical experiments for the Darcy flow problem showed that the barycentric Hodge star operator reproduced the second order convergence rate attained through the circumcentric (diagonal) Hodge star operator. Other experiments for the incompressible Navier-Stokes equations showed similar convergence rates for the barycentric versus the circumcentric Hodge star operators. The convergence was of a first order rate for the unstructured meshes and of a second order rate for structured-triangular simplicial meshes. Furthermore, a superconvergence behavior, of a rate close to second order, was observed when using the barycentric Hodge star operator on unstructured meshes generated through sequential mesh subdivision of a coarser mesh. This super-convergence was attributed to the subdivision process itself which introduced new primal edges that satisfy the second order convergence condition. In 


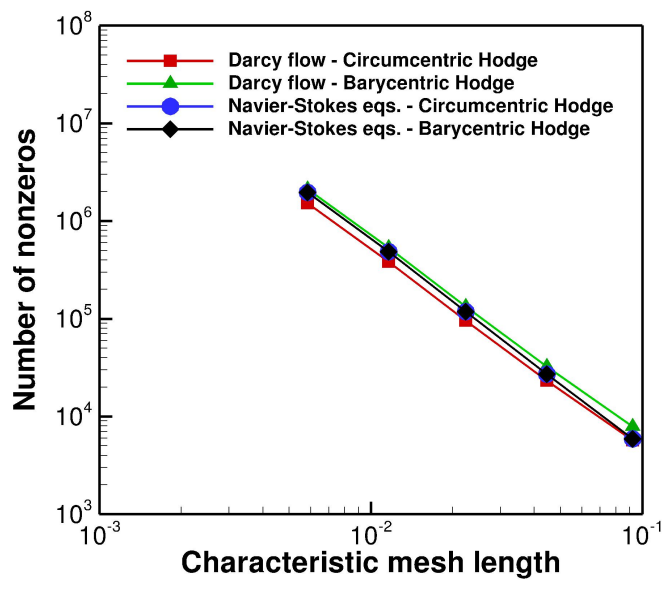

(a)

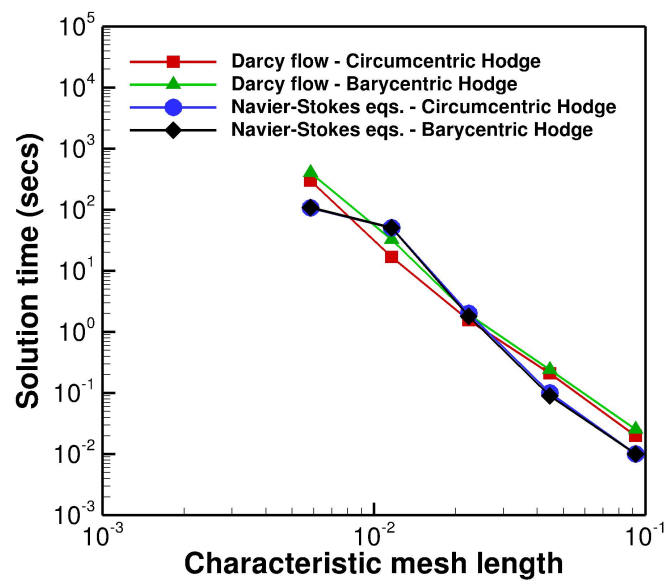

(b)

Figure 7: (a) The number of non-zeros in the global matrices. (b) The solution time (in seconds) for various mesh sizes.

all the conducted experiments, the Galerkin and the barycentric Hodge operators had almost identical results. Furthermore, both the Galerkin and the barycentric Hodge operators could reproduce the expected convergence order even for highly distorted non-Delaunay meshes.

With regards to the computational cost aspects, the barycentric Hodge operator had a number of nonzero entries almost five times as the circumcentric Hodge operator. However, due to the multiplications by the exterior derivative matrices during the numerical solution, the resulting stiffness matrix for the Darcy flow problem had only $\sim 40 \%$ increase in the number of nonzero entries when using the barycentric Hodge operator, in comparison to the circumcentric Hodge operator case. Furthermore, the number of nonzero entries in the incompressible Navier-Stokes system matrix did not increase due to the usage of the barycentric Hodge operator. The same results apply also to the Galerkin Hodge operator, which has a sparsity structure identical to the barycentric Hodge operator. The modest increase in the computational cost, the admission of arbitrary simplicial and even highly distorted meshes, in addition to the super-convergence attainable through mesh subdivision suggest that the barycentric Hodge operators can be favorable over the circumcentric Hodge operators for DEC implementations over surfaces, as long as the $*_{1}^{-1}$ is not required. However, the circumcentric Hodge star operators continue to have some advantages due to their diagonal structure, which facilitates the definition of the inverse operators in all cases.

Finally, a potential theoretical advantage of the barycentric Hodge star is a pathway towards exploring a connection between DEC and finite element exterior calculus due to the quadrature relationship noted in Section 3. Characterization of a relationship between dual meshes, primal interpolation and the resulting discrete Hodge star would also be of interest for future work.

\section{Acknowledgments}

This research was supported by the KAUST Office of Competitive Research Funds under Award No. URF/1/1401-01-01. The work of ANH was supported in part by NSF Grant No. CCF-1064429.

\section{References}

[1] A. N. Hirani, Discrete exterior calculus, Ph.D. thesis, California Institute of Technology (2003).

[2] M. Desbrun, A. N. Hirani, M. Leok, J. E. Marsden, Discrete exterior calculus, arXiv preprint math/0508341.

[3] A. N. Hirani, K. B. Nakshatrala, J. H. Chaudhry, Numerical method for Darcy flow derived using Discrete Exterior Calculus, Int. J. Comput. Meth. Eng. Sci. Mech. 16 (3) (2015) 151-169. doi:10.1080/15502287.2014.977500.

[4] A. Gillette, C. Bajaj, Dual formulations of mixed finite element methods with applications, Comput. Aided Design 43 (10) (2011) 1213-1221.

[5] S. Elcott, Y. Tong, E. Kanso, P. Schröder, M. Desbrun, Stable, circulationpreserving, simplicial fluids, ACM Trans. Graph. 26 (1) (2007) 4.

[6] P. Mullen, K. Crane, D. Pavlov, Y. Tong, M. Desbrun, Energy-preserving integrators for fluid animation, ACM Trans. Graph. 28 (3) (2009) 38.

[7] M. S. Mohamed, A. N. Hirani, R. Samtaney, Discrete exterior calculus discretization of incompressible Navier-Stokes equations over surface simplicial meshes, J. Comput. Phys. 312 (2016) 175-191. doi:10.1016/j.jcp.2016.02.028.

[8] D. Pavlov, P. Mullen, Y. Tong, E. Kanso, J. E. Marsden, M. Desbrun, Structure-preserving discretization of incompressible fluids, Physica D: Nonlinear Phenomena 240 (6) (2011) 443-458.

[9] E. Gawlik, P. Mullen, D. Pavlov, J. Marsden, M. Desbrun, Geometric, variational discretization of continuum theories, Physica D: Nonlinear Phenomena 240 (21) (2011) 1724 - 1760. doi:10.1016/j.physd.2011.07.011.

[10] O. Azencot, S. Weißmann, M. Ovsjanikov, M. Wardetzky, M. Ben-Chen, Functional fluids on surfaces, Computer Graphics Forum 33 (5) (2014) 237-246. doi:10.1111/cgf.12449.

[11] O. Azencot, M. Ovsjanikov, F. Chazal, M. Ben-Chen, Discrete derivatives of vector fields on surfaces-an operator approach, ACM Trans. Graph. 34 (3) (2015) 29.

[12] B. Liu, G. Mason, J. Hodgson, Y. Tong, M. Desbrun, Model-reduced variational fluid simulation, ACM Trans. Graph. 34 (6) (2015) 244:1-244:12. doi: $10.1145 / 2816795.2818130$.

[13] A. N. Hirani, K. Kalyanaraman, E. B. VanderZee, Delaunay Hodge star, Comput. Aided Design 45 (2) (2013) 540-544. 
[14] A. Bossavit, Computational electromagnetism: variational formulations, complementarity, edge elements, Academic Press, 1998.

[15] F. Trevisan, L. Kettunen, Geometric interpretation of discrete approaches to solving magnetostatic problems, IEEE T. Magn. 40 (2) (2004) 361365.

[16] B. Auchmann, S. Kurz, A geometrically defined discrete Hodge operator on simplicial cells, IEEE T. Magn. 42 (4) (2006) 643-646.

[17] M. Fisher, B. Springborn, P. Schröder, A. I. Bobenko, An algorithm for the construction of intrinsic Delaunay triangulations with applications to digital geometry processing, Computing 81 (2-3) (2007) 199-213.

[18] H. Whitney, Geometric Integration Theory, Princeton University Press, Princeton, N. J., 1957.

[19] J. Warren, S. Schaefer, A. N. Hirani, M. Desbrun, Barycentric coordinates for convex sets, Adv. Comput. Math. 27 (3) (2007) 319-338.

[20] D. Arnold, R. Falk, R. Winther, Finite element exterior calculus: from Hodge theory to numerical stability, B. Am. Math. Soc. 47 (2) (2010) 281-354.

[21] R. A. Nicolaides, Direct discretization of planar div-curl problems, SIAM J. Numer. Anal. 29 (1) (1992) 32-56. 